\title{
Defect analysis of star defects in GaN thin films grown on HVPE GaN substrates
}

Tim Ruggles ${ }^{1}$, Julia Deitz ${ }^{2}$, Andrew Allerman ${ }^{1}$, C. Barry Carter ${ }^{3}$ and Joseph Michael ${ }^{2}$

${ }^{1}$ Sandia National Laboratories, Albuquerque, New Mexico, United States, ${ }^{2}$ Sandia National Laboratory, United States, ${ }^{3}$ University of Connecticut, Connecticut, United States

The potential of GaN-based power electronics is limited by defects, most of which result from lattice parameter mismatch with the substrate. Use of GaN substrates manufactured with halide-vapor phase epitaxy (HVPE) reduces these defects but can introduce different defects. This study investigates one such defect, the star defect, recently reported in the literature [1]. These defects are hundreds of microns in size and may be related to defects reported in $\mathrm{GaN}$ substrates [2]. Understanding the structure of these defects is crucial to developing growth processes to prevent them or mitigate their effect on device performance. The films investigated were deposited with metal-organic vapor phase deposition (MOVPE) to 10 microns in thickness. The film was n-doped to $5 \times 10^{16} \mathrm{~cm}^{-3}$ net electron concentration.

The star defects in the film were investigated using a combination of two scanning electron microscopy techniques, electron channeling contrast imaging (ECCI) and high-resolution electron backscatter diffraction (HREBSD). ECCI was used to detect the defects and probe their abundance across the film, while HREBSD was using to identify the smaller defects comprising the star defects using a novel variant of Nye-Kröner analysis. The star defects appear as intersections of straight lines on the surface aligned with the $<10-10>$ directions hundreds of microns in length. HREBSD analysis shows that these defects are in fact low-angle tilt boundaries made up of regular arrays of < a >-type pyramidal edge dislocations spaced between 100 and $200 \mathrm{~nm}$ apart, with each ray having a different Burgers vector in the family. In addition to the low-angle tilt boundaries the "rays" of the star defect are composed of, the "cores" were also composed of unique defects, including a significant amount of threading dislocations. Unlike the rays of the star defects, which varied only in length, the cores have significant variability (see Figure 1 for an example of a star defect core).

This paper describes objective technical results and analysis. Any subjective views or opinions that might be expressed in the paper do not necessarily represent the views of the U.S. Department of Energy or the United States Government. Sandia National Laboratories is a multimission laboratory managed and operated by National Technology and Engineering Solutions of Sandia, LLC., a wholly owned subsidiary of Honeywell International, Inc., for the U.S. Department of Energy's National Nuclear Security Administration under contract DE-NA-0003525. 


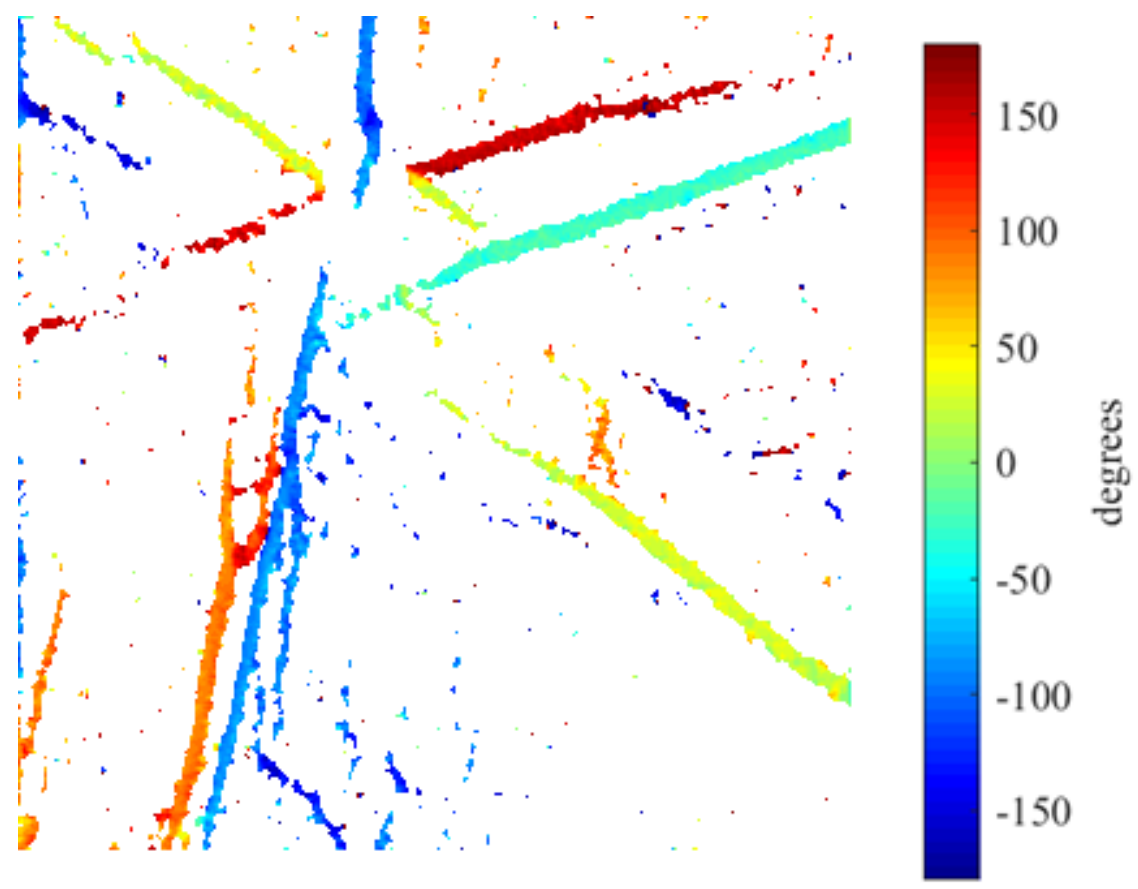

Figure 1. A map of the angle of Burgers vector with respect to the crystallographic reference frame of the core of a star defect. Regions with low signal (where it is presumed there is no defect content) were blanked out for visual clarity. The scan is 24 microns square.

\section{References}

[1] Ruggles, T., Deitz, J., Allerman, A., Carter, C.B., Michael, J., 2021, Identification of star defects in gallium nitride with HREBSD and ECCI. Microscopy \& Microanalysis, in press

[2] Raghothamachar, B., Liu, Y., Peng, H., Ailihuamaer, T., Dudley, M., Shahedipour-Sandvik, F.S., Jones, K.A., Armstrong, A., Allerman, A.A., Han, J., et al., 2020. X-ray topography characterization of gallium nitride substrates for power device development. Journal

of Crystal Growth , 125709. 